\title{
Invest early and often
}

\section{Funding organizations need to support young investigators to ensure the future of interdisciplinary research.}

In 2007, the US National Institutes of Health (NIH) launched a policy to reverse an alarming decline in awards to new investigators (that is, applicants having received no prior independent funding via a major mechanism; http://grants.nih. gov/grants/new_investigators/). Although this policy has improved the number and percentage of awards for new investigators (from $23.9 \%$ in 2008 to $31.8 \%$ in 2010), the average age at which a new investigator (with a PhD) receives his or her first R01 award remains 42 (http://nexus.od.nih.gov/ all/2012/02/13/age-distribution-of-nihprincipal-investigators-and-medical-schoolfaculty/). Newer disciplines such as chemical biology that are largely populated by young scientists are disproportionately negatively affected by this funding environment.

Many factors contribute to the increasing age of new investigators. Scientific research grows ever more complex, and innovative work often requires an interdisciplinary approach, which often prolongs training for students and postdocs. The fact that a growing percentage of established investigators remain active in research beyond the traditional retirement age (Nature 483, 233-235, 2012) translates into fewer opportunities for young scientists. This trend and other institutional barriers that increase the age of new faculty also decrease the pool of young scientists who are even eligible to apply for funding. The emphasis placed on experience and preliminary results by grant review panels, which generally do not contain young investigators, are inherent disadvantages that can also prolong the time it takes for young investigators to obtain their first grants.

Regardless of the origin of these trends, the current state of funding is detrimental to the present and future of scientific research. Consider that from 1980 to 2010, the 96 winners of the Nobel Prize were on average 41 years old when they performed their award-winning research (PLoS One 6, e29738, 2011), and, in general, most investigators do their most influential work mid-career ( $36-45$ years old; Scientometrics 61, 1117-1128, 2004). We suggest that the time for small adjustments in research funding philosophy or strategy to promote the careers of young scientists has passed. Rather, it is time for dramatic action; major funding bodies should consider allocating funds for applicants at particular stages of their career, and applicant pools should be considered individually by career stage. The NIH could also consider alternative models championed by agencies such as the Howard Hughes Medical Institute (http://www.hhmi. org/research/index.html) or the European Research Council (http://erc.europa.eu/ about-erc/facts-and-figures) by investing in people as opposed to projects and providing long-term support so investigators can tackle more challenging problems. Notably, both of these agencies have funding mechanisms targeting young investigators who are considered against a pool of their peers.

Funding agencies such as the NIH have more power to improve the plight of young investigators than any academic center, university president or established investigator. Accordingly, if the NIH were to adopt and enforce policies and funding initiatives that incentivized institutions to hire young scientists, barriers outside of the direct purview of the NIH could disappear. Research institutions will make the necessary changes to comply with such policies to maintain their funding streams. In the absence of this type of disruptive innovation, the future of young disciplines such as chemical biology and, more broadly, of interdisciplinary science is in jeopardy.

\section{Let's connect}

\section{Social media offer rapid and informal mechanisms to exchange ideas and build ties within the community.}

Editorials at Nature Chemical Biology often end with a question or call to action: How can we best define the scientific scope of a single paper? How can small groups achieve big goals? Celebrate synthesis! Writing these editorials, as well as research highlights and the occasional journalistic article, gives us the opportunity to raise important issues and highlight content of relevance to the field. However, we also use these pieces to seek community feedback about central issues in chemical biology and our journal policies. To facilitate communication with improved timeliness and with the widest possible reach, Nature Chemical Biology is now on Twitter and Facebook.

Online media are changing the way we communicate. We previously highlighted the increased speed at which scientists communicate via blogs and other online newsfeeds, particularly to discuss primary research papers (Nat. Chem. Biol. 7, 65, 2011; Nat. Chem. Biol. 5, 601, 2009). However, scientific discourse consists of more than just a collection of articles, and many of the announcements and highlights that serve to connect the community are themselves well served by the informal tone and immediacy of online venues.

To take advantage of these online opportunities, we are using Twitter (@nchembio) and Facebook (http://on.fb. me/GWF4hb) to highlight content published in the journal as well as to draw attention to papers published elsewhere in the literature, topical news stories and opportunities in the field. We are linking to relevant discussions about scientific publishing and to conferences where you can meet our editors. On our Twitter feed, we also feature tweets identified by the editors' initials, allowing readers to get a broader perspective of specific editors' interests.

Perhaps most importantly, Twitter and Facebook provide a more casual forum to share your thoughts with the journal and the community at large, lowering the barriers to communication that typically only occurs at conferences or via individual contacts. After all, we best serve the community when we are informed about questions and concerns shared by scientists in the field.

We hope that you will find our social media content informative and engaging. Similarly, we encourage you to take part in the growing community by sharing your perspective and providing feedback on editorials or other projects at the journal. Thus, your call to action: Follow us on Twitter, like us on Facebook and keep us posted. 